\title{
ON THE $k$-BUCHSBAUM PROPERTY OF POWERS OF STANLEY-REISNER IDEALS
}

\author{
NGUYÊN CÔNG MINH AND YUKIO NAKAMURA
}

\begin{abstract}
Let $S=K\left[x_{1}, x_{2}, \ldots, x_{n}\right]$ be a polynomial ring over a field $K$. Let $\Delta$ be a simplicial complex whose vertex set is contained in $\{1,2, \ldots, n\}$. For an integer $k \geq 0$, we investigate the $k$-Buchsbaum property of residue class rings $S / I^{(t)}$ and $S / I^{t}$ for the Stanley-Reisner ideal $I=I_{\Delta}$. We characterize the $k$-Buchsbaumness of such rings in terms of the simplicial complex $\Delta$ and the power $t$. We also give a characterization in the case where $I$ is the edge ideal of a simple graph.
\end{abstract}

\section{$\S 1$. Introduction}

Let $S=K\left[x_{1}, x_{2}, \ldots, x_{n}\right]$ be a polynomial ring over a field $K$ with the maximal ideal $\mathfrak{m}=\left(x_{1}, x_{2}, \ldots, x_{n}\right)$. Let $\Delta$ be a simplicial complex on a vertex set contained in $[n]=\{1,2, \ldots, n\}$. Let $I_{\Delta}$ be the Stanley-Reisner ideal of $\Delta$ in $S$. Let $k$ be a nonnegative integer. For an ideal $J$ in $S$, we say that $S / J$ is $k$-Buchsbaum if $\mathfrak{m}^{k} H_{\mathfrak{m}}^{i}(S / J)=(0)$ for all $i<\operatorname{dim} S / J$, where $H_{\mathfrak{m}}^{i}(S / J)$ is the $i$ th local cohomology module of $S / J$ with respect to $\mathfrak{m}$. Obviously, the 0-Buchsbaum property implies the Cohen-Macaulay property, and the 1-Buchsbaum property implies the quasi-Buchsbaum property. The purpose of this paper is to investigate the $k$-Buchsbaum property of the residue class rings $S / I_{\Delta}^{t}$ and $S / I_{\Delta}^{(t)}$, where $I_{\Delta}^{(t)}$ stands for the th symbolic power of $I_{\Delta}$. The first main result of this paper is the following.

Theorem 3.2. Let $I=I_{\Delta}$ be the Stanley-Reisner ideal of a simplicial complex $\Delta$. Let $k$ be a nonnegative integer. Then, the following statements are equivalent:

Received October 6, 2010. Revised January 3, 2013. Accepted April 2, 2013.

First published online December 17, 2013.

2010 Mathematics Subject Classification. Primary 13H10; Secondary 13F55, 05 E99.

Minh's work was partially supported by the Japan Society for the Promotion of Science Ronpaku (Ph.D. dissertation) program and by Vietnam National Foundation for Science and Technology Development grant 101.01-2011.08.

(C) 2014 by The Editorial Board of the Nagoya Mathematical Journal 
(1) $S / I^{(t)}$ is Cohen-Macaulay for all $t \geq 1$;

(2) $S / I^{(t)}$ is $k$-Buchsbaum for all $t \geq 1$;

(3) $S / I^{(t)}$ is $k$-Buchsbaum for some $t \geq k+3$;

(4) $\Delta$ is a matroid.

The notion of a matroid is a concept of discrete mathematics (for its definition, see the text following Lemma 2.5). That is a quite broad generalization of linear independence and has widespread applications, for example, to graph theory. The second main result of the paper regards the ordinary powers of the Stanley-Reisner ideal, as follows.

COROLlary 4.7. Let $I=I_{\Delta}$ be the Stanley-Reisner ideal of a simplicial complex $\Delta$. Let $k$ be a nonnegative integer. Then, the following statements are equivalent:

(1) $S / I^{t}$ is Cohen-Macaulay for all $t \geq 1$;

(2) $S / I^{t}$ is $k$-Buchsbaum for all $t \geq 1$;

(3) $S / I^{t}$ is $k$-Buchsbaum for some $t \geq k+3$;

(4) $I$ is a complete intersection.

This is a combination of the results of a 1-dimensional case (Theorem 4.6) and a higher-dimensional case (Theorem 4.4).

Research on the Cohen-Macaulay property of $S / I_{\Delta}^{(t)}$ and $S / I_{\Delta}^{t}$ was begun by [MT1] and [GH] for 1-dimensional simplicial complexes $\Delta$. Then, by the authors, the Buchsbaum properties of $S / I_{\Delta}^{(t)}$ and $S / I_{\Delta}^{t}$ were studied in [MN1] and [MN2] for $\Delta$ with $\operatorname{dim} \Delta=1$. Moreover, the $k$-Buchsbaum property of $S / I_{\Delta}^{(t)}$ was studied in [MN3]. Later, the Cohen-Macaulay property of $S / I_{\Delta}^{(t)}$ for an arbitrary dimension was studied in [MT2] and [V], and the Buchsbaum cases were studied in [TT].

On the other hand, according to [CN, Corollary], the Cohen-Macaulayness of $S / I_{\Delta}^{t}$ for all $t>0$ implies that $I_{\Delta}$ is a complete intersection. So, it is interesting to research the properties of $S / I_{\Delta}$ or $I_{\Delta}$ under the condition that $S / I_{\Delta}^{t}$ has good properties for every large-enough $t$. One can see various results in [GT], [TY], [RTY], and [TT]. The authors of these papers developed many kinds of properties for $S / I_{\Delta}$ or $I_{\Delta}$ under the condition that $S / I_{\Delta}^{t}$ or $S / I_{\Delta}^{(t)}$ is Cohen-Macaulay, Buchsbaum, generalized CohenMacaulay, or $\left(S_{2}\right)$ for every large-enough $t$; also, they determined the range for such a $t$. The target of this paper is the $k$-Buchsbaumness of $S / I_{\Delta}^{t}$ and $S / I_{\Delta}^{(t)}$. One of the interesting points is the discovery of the relation between $t$ and $k$. 
For a simple graph $G$, we have a square-free monomial ideal $I(G)$ in $S$ which is called the edge ideal of $G$. An edge ideal can be expressed as the Stanley-Reisner ideal of a suitable simplicial complex; hence, the result for Stanley-Reisner ideals can be applied to the case of edge ideals. In Corollary 3.7, we give a characterization for $S / I(G)^{(t)}$ to be $k$-Buchsbaum for every large-enough $t$ in terms of $G$.

This paper consists of four sections. In Section 2, we set up the fundamental notation and terminologies, for which we mainly refer to the book $[\mathrm{BH}]$. Degree complex $\Delta_{\mathbf{a}}(I)$ plays an important role (for details, we refer to $[\mathrm{T}]$, [MN1], and [MN2]). In Section 3, we provide an argument for the symbolic powers of Stanley-Reisner ideals. Section 4 is devoted to the argument of ordinary powers.

\section{§2. Preliminaries}

A simplicial complex $\Delta$ on $[n]:=\{1,2, \ldots, n\}$ is a collection of subsets of $[n]$ such that $F \in \Delta$ whenever $F \subseteq F^{\prime}$ for some $F^{\prime} \in \Delta$. Here $F \in \Delta$ is called a face of $\Delta$. We put $\operatorname{dim} F=|F|-1$, where $|F|$ is the cardinality of $F$, and we put $\operatorname{dim} \Delta=\max \{\operatorname{dim} F \mid F \in \Delta\}$, which is called the dimension of $\Delta$.

Let $K$ be a field, and let $S=K\left[x_{1}, x_{2}, \ldots, x_{n}\right]$ be a polynomial ring of $n$ variables over $K$. We denote the homogeneous maximal ideal of $S$ by $\mathfrak{m}=\left(x_{1}, x_{2}, \ldots, x_{n}\right)$. The Stanley-Reisner ideal of $\Delta$ is defined as

$$
I_{\Delta}=\left(\prod_{i \in F} x_{i} \mid F \notin \Delta\right)=\bigcap_{F \in \operatorname{Max}(\Delta)} P_{F},
$$

where $P_{F}$ is the ideal in $S$ generated by $\left\{x_{j} \mid j \notin F\right\}$.

Each element in $\operatorname{Max}(\Delta)$ is called a facet, which is a maximal face of $\Delta$ with respect to inclusion, and the intersection of $P_{F}$ s gives an irredundant primary decomposition of $I_{\Delta}$. If all facets of $\Delta$ have the same dimension, we say that $\Delta$ is pure. Every square-free monomial ideal of $S$ can be written as a Stanley-Reisner ideal of a suitable simplicial complex. The residue class ring $K[\Delta]=S / I_{\Delta}$ is called the Stanley-Reisner ring of $\Delta$. It is known that $\operatorname{dim} K[\Delta]=\operatorname{dim} \Delta+1$.

Let $\mathbf{a}=\left(a_{1}, a_{2}, \ldots, a_{n}\right) \in \mathbb{Z}^{n}$. We put the subset $G_{\mathbf{a}}=\left\{i \mid a_{i}<0\right\}$ of $[n]$, and we write $\mathbf{x}^{\mathbf{a}}=\prod_{j=1}^{n} x_{j}^{a_{j}}$, which is an element of $S_{G_{\mathbf{a}}}$, where $S_{G_{\mathbf{a}}}$ is the localization $S\left[x_{i}^{-1} \mid i \in G_{\mathbf{a}}\right]$ of $S$.

For a monomial ideal $I$ of $S$, the degree complex $\Delta_{\mathbf{a}}(I)$ is the simplicial complex on $[n]$ defined as follows. 
Definition 2.1. Let $I$ be a monomial ideal of $S$ (see $[\mathrm{T}]$ ). Let $\mathbf{a} \in \mathbb{Z}^{n}$. Then $\Delta_{\mathbf{a}}(I)$ is a collection of subsets, $F$ of $[n]$, satisfying the following two conditions:

(1) $F \cap G_{\mathbf{a}}=\emptyset$;

(2) for every minimal monomial generator $\mathbf{x}^{\mathbf{b}}$ of $I$, where $\mathbf{b}=\left(b_{1}, b_{2}, \ldots, b_{n}\right)$, there exists an index $i \notin F \cup G_{\mathbf{a}}$ with $b_{i}>a_{i}$.

We note that the second condition is equivalent to saying that $\mathbf{x}^{\mathbf{a}} \notin$ $I S_{F \cup G_{\mathbf{a}}}$. The degree complex is a useful tool to describe the local cohomology modules of $S / I$. We denote by $\widetilde{H}^{j}(\Delta ; K)$ the reduced cohomology group of a simplicial complex $\Delta$ over $K$ (see [BH, Section 5.3]).

Theorem 2.2 ([T, Lemma 2]). Let I be a monomial ideal of $S$. For each $p \in \mathbb{Z}$ and $\mathbf{a} \in \mathbb{Z}^{n}$, there is an isomorphism of $K$-vector spaces:

$$
H_{\mathfrak{m}}^{p}(S / I)_{\mathbf{a}} \cong \widetilde{H}^{p-\left|G_{\mathbf{a}}\right|-1}\left(\Delta_{\mathbf{a}}(I) ; K\right) .
$$

For a Stanley-Reisner ideal $I_{\Delta}$ and a positive integer $r$, the $r$ th symbolic power of $I_{\Delta}$ is given as $\bigcap_{F \in \operatorname{Max}(\Delta)} P_{F}{ }^{r}$, which is also a monomial ideal. The following lemma is very useful for calculating the degree complex of symbolic powers of $I_{\Delta}$. For $\mathbf{a}=\left(a_{1}, a_{2}, \ldots, a_{n}\right) \in \mathbb{N}^{n}$ and $F \in[n]$, we put $\sigma_{F}^{\mathbf{a}}=\sum_{i \notin F} a_{i}$.

LEMma 2.3 ([MT2, Lemma 1.5]). Let $I=I_{\Delta}$ be the Stanley-Reisner ideal of $\Delta$. Let $\mathbf{a}=\left(a_{1}, a_{2}, \ldots, a_{n}\right) \in \mathbb{N}^{n}$. Suppose that $I$ is unmixed (i.e., that $\Delta$ is pure). Then, for a positive integer $r, \operatorname{Max}\left(\Delta_{\mathbf{a}}\left(I^{(r)}\right)\right)$ consists of $F \in \operatorname{Max}(\Delta)$ satisfying $\sigma_{F}^{\mathbf{a}}<r$.

For a simplicial complex $\Delta$ and $F \in \Delta$, we define two subcomplexes:

$$
\begin{aligned}
& \operatorname{link}_{\Delta} F=\{H \in \Delta \mid H \cap F=\emptyset, H \cup F \in \Delta\}, \\
& \operatorname{star}_{\Delta} F=\{H \in \Delta \mid H \cup F \in \Delta\} .
\end{aligned}
$$

Note that a degree complex can be written as a link. The proof of the following lemma is given in $[\mathrm{M}]$ and [MT2]. For convenience, we provide a brief proof below.

LEMMA 2.4 ([MT2, Lemma 1.5]). Let I be a monomial ideal of S. For $\mathbf{a} \in \mathbb{Z}^{n}$, we define $\mathbf{a}_{+} \in \mathbb{N}^{n}$ so that

$$
\begin{aligned}
& \left(\mathbf{a}_{+}\right)_{j}= \begin{cases}a_{j} & \left(a_{j} \geq 0\right), \\
0 & (\text { otherwise }) .\end{cases} \\
& \text { If } \Delta_{\mathbf{a}}(I) \neq \emptyset, \text { then } \Delta_{\mathbf{a}}(I)=\operatorname{link}_{\Delta_{\mathbf{a}_{+}}(I)} G_{\mathbf{a}} .
\end{aligned}
$$


Proof. Note that $\Delta_{\mathbf{a}}(I) \neq \emptyset$ (i.e., that $\left.\emptyset \in \Delta_{\mathbf{a}}(I)\right)$ if and only if $G_{\mathbf{a}} \in$ $\Delta_{\mathbf{a}_{+}}(I)$. Let $F \in \Delta_{\mathbf{a}}(I)$. Then, $\mathbf{x}^{\mathbf{a}} \notin I S_{F \cup G_{\mathbf{a}}}$, which is equivalent to saying that $\mathbf{x}^{\mathbf{a}+} \notin I S_{F \cup G_{\mathbf{a}}}$. Hence, $F \cap G_{\mathbf{a}}=\emptyset$ and $F \cup G_{\mathbf{a}} \in \Delta_{a_{+}}(I)$, which implies that $F \in \operatorname{link}_{\Delta_{\mathbf{a}_{+}(I)}} G_{\mathbf{a}_{\mathbf{a}}}$. The converse implication follows from the same argument.

The following lemma is proved in [MN1, Lemma 2.3] in the case where $\mathbf{a}, \mathbf{b} \in \mathbb{N}^{n}$. We note that the proof also works in the case where $\mathbf{a} \in \mathbb{Z}^{n}$, $\mathbf{b} \in \mathbb{N}^{n}$ with $G_{\mathbf{a}}=G_{\mathbf{a}+\mathbf{b}}$.

Lemma 2.5 ([MN1, Lemma 2.3]). Let I be a monomial ideal of $S$. Let $\mathbf{a} \in \mathbb{Z}^{n}$, and let $\mathbf{b} \in \mathbb{N}^{n}$, with $G_{\mathbf{a}}=G_{\mathbf{a}+\mathbf{b}}$. Then, for any integers $j \geq 0$, we have the following commutative diagram:

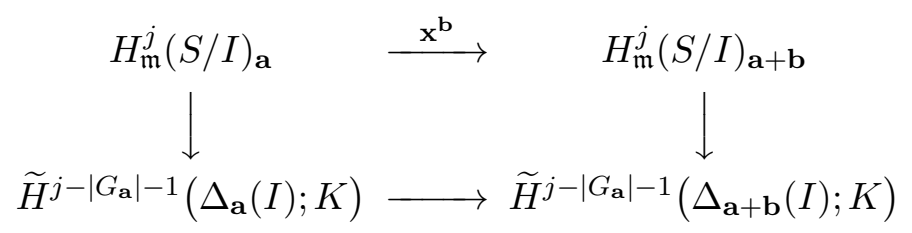

where the vertical maps are isomorphisms as in Theorem 2.2, the top map is induced from the multiplicative map $S / I \ni f \mapsto \mathbf{x}^{\mathbf{b}} f \in S / I$, and the bottom map is induced from the natural embedding $\Delta_{\mathbf{a}+\mathbf{b}}(I) \subseteq \Delta_{\mathbf{a}}(I)$ of simplicial complexes.

A simplicial complex $\Delta \neq \emptyset$ is called a matroid if the following condition is satisfied: for $F, G \in \Delta$ with $|F|<|G|$, there exists $x \in G \backslash F$ such that $F \cup\{x\} \in \Delta$.

We note that the following lemma is useful in checking for whether a simplicial complex is a matroid.

Lemma 2.6 ([S, Theorem 39.1]). A simplicial complex $\Delta \neq \emptyset$ is a matroid if and only if, for any $F, G \in \Delta$ with $|F \backslash G|=1$ and $|G \backslash F|=2$, there exists $x \in G \backslash F$ such that $F \cup\{x\} \in \Delta$.

A simple graph $G$ consists of a finite set $V(G)$ of vertices and a collection of edges $E(G)$, which are 2-element subsets of $V(G)$. We note that a simple graph has no loops and no parallels. In this article, we always assume that a graph $G$ is simple and that $V(G) \subseteq[n]$. The edge ideal of $G$ is the ideal of $S$ generated by $\left\{x_{i} x_{j} \mid\{i, j\} \in E(G)\right\}$, denoted by $I(G)$. Note that an edge ideal is a square-free monomial ideal, so it can be written as a StanleyReisner ideal of a suitable simplicial complex. 


\section{$\S 3$. Symbolic powers}

The following proposition is a key component of this paper. Let $\mathbf{e}_{i} \in \mathbb{Z}^{n}$ be the $i$ th unit vector. For a subset $F$ of $[n]$, we put $\mathbf{e}_{F}=\sum_{i \in F} \mathbf{e}_{i}$.

Proposition 3.1. Let $\Delta$ be a pure simplicial complex on $[n]$. We put $I=I_{\Delta}$. Let $k$ and $t$ be integers such that $k \geq 0$ and $t \geq k+3$. If $\Delta$ is not a matroid, then $\operatorname{dim} S / I^{(t)}>0$ and $\mathfrak{m}^{k} H_{\mathfrak{m}}^{r}\left(S / I^{(t)}\right) \neq(0)$ for some $r<$ $\operatorname{dim} S / I^{(t)}$.

Proof. By Lemma 2.6, we can choose $F_{1}, F_{2} \in \Delta$ such that $F_{1} \backslash F_{2}=$ $\{i\}, F_{2} \backslash F_{1}=\{j, p\}, F_{1} \cup\{j\} \notin \Delta$, and $F_{1} \cup\{p\} \notin \Delta$. Let $L=\operatorname{link}_{\Delta}\{i\} \cap$ $\operatorname{link}_{\Delta}\{j, p\}$. Note that $F_{1} \cap F_{2} \in L$, so we can take $F \in \operatorname{Max}(L)$ such that $F_{1} \cap F_{2} \subseteq F$. Let $\mathbf{a}=(t-1) \mathbf{e}_{i}+\mathbf{e}_{j}+\mathbf{e}_{p}-\mathbf{e}_{F}$. In particular, $\mathbf{a}_{+}=(t-1) \mathbf{e}_{i}+$ $\mathbf{e}_{j}+\mathbf{e}_{p}$ and $G_{\mathbf{a}}=F$. Then, one can check that

$$
\Delta_{\mathbf{a}_{+}}\left(I^{(t)}\right)=\operatorname{star}_{\Delta}\{i\} \cup \operatorname{star}_{\Delta}\{j, p\}
$$

In fact, $H \in \operatorname{Max}\left(\Delta_{\mathbf{a}_{+}}\left(I^{(t)}\right)\right)$ if and only if $\sigma_{H}^{\mathbf{a}_{+}}<t$ and $H \in \operatorname{Max}(\Delta)$ by Lemma 2.3. On the other hand, it is easy to see that the inequality $\sigma_{H}^{\mathbf{a}_{+}}<t$ is equivalent to saying that $i \in H$ or $j, p \in H$. Therefore, it follows that $H \in \operatorname{Max}\left(\Delta_{\mathbf{a}_{+}}\left(I^{(t)}\right)\right)$ if and only if $H \in \operatorname{Max}\left(\operatorname{star}_{\Delta}\{i\} \cup \operatorname{star}_{\Delta}\{j, p\}\right)$. Thus, the equality holds true. By Lemma 2.4, we get

$$
\begin{aligned}
\Delta_{\mathbf{a}}\left(I^{(t)}\right) & =\operatorname{link}_{\Delta_{\mathbf{a}_{+}}\left(I^{(t)}\right)} G_{\mathbf{a}} \\
& =\operatorname{link}_{\mathrm{star}_{\Delta}\{i\} \cup \operatorname{star}_{\Delta}\{j, p\}} F \\
& =\operatorname{link}_{\operatorname{star}_{\Delta}\{i\}} F \cup \operatorname{link}_{\operatorname{star}_{\Delta}\{j, p\}} F .
\end{aligned}
$$

Here, we note that since $\{i\} \in \operatorname{link}_{\operatorname{star}_{\Delta}\{i\}} F$ and $\{j, p\} \in \operatorname{link}_{\operatorname{star}_{\Delta}\{j, p\}} F$, both links contain at least a vertex. Furthermore, one can show that $\operatorname{link}_{\text {star }_{\Delta}\{i\}} F \cap \operatorname{link}_{\text {star }_{\Delta}\{j, p\}} F=\{\emptyset\}$. In fact, suppose that there is a vertex $x \in[n]$ such that $\{x\} \in \operatorname{link}_{\text {star }_{\Delta}\{i\}} F \cap \operatorname{link}_{\operatorname{star}_{\Delta}\{j, p\}} F$. Then, it follows that $x \notin F, F \cup\{x\} \in \operatorname{star}_{\Delta}\{i\}$, and $F \cup\{x\} \in \operatorname{star}_{\Delta}\{j, p\}$. In other words, $x \notin F$, $F \cup\{x, i\} \in \Delta$, and $F \cup\{x, j, p\} \in \Delta$. Note that $x$ is different from $i, j$, and $p$. In fact, if $x=j$, then $F \cup\{i, j\} \in \Delta$, whence $F_{1} \cap F_{2} \cup\{i, j\}=F_{1} \cup\{j\} \in \Delta$, which is a contradiction.

From the same argument, it follows that $x \neq p$ and $x \neq i$, too. Therefore, $F \cup\{x\} \in \operatorname{link}_{\Delta}\{i\}$ and $F \cup\{x\} \in \operatorname{link}_{\Delta}\{j, p\}$. Consequently, we have $F \cup$ $\{x\} \in L$, which contradicts the maximality of $F$ in $L$. Hence, $\operatorname{link}_{\operatorname{star}_{\Delta}\{i\}} F \cap$ 
$\operatorname{link}_{\operatorname{star}_{\Delta}\{j, p\}} F=\{\emptyset\}$. Thus, we conclude that $\Delta_{\mathbf{a}}\left(I^{(t)}\right)$ is the disjoint union of nonempty simplicial complexes $\operatorname{link}_{\operatorname{star}_{\Delta}\{i\}} F$ and $\operatorname{link}_{\text {star }_{\Delta}\{j, p\}} F$. In particular, $\Delta_{\mathbf{a}}\left(I^{(t)}\right)$ has at least two connected components. Let $r=|F|+1$. Since $\operatorname{dim} F \leq \operatorname{dim}_{\operatorname{link}}\{j, p\} \leq \operatorname{dim} \Delta-2, \quad r=\left|G_{\mathbf{a}}\right|+1 \leq \operatorname{dim} \Delta<$ $\operatorname{dim} S / I^{(t)}$. Thanks to the formula of Theorem 2.2 , we get

$$
\begin{aligned}
H_{\mathfrak{m}}^{r}\left(S / I^{(t)}\right)_{\mathbf{a}} & =\widetilde{H}^{r-\left|G_{\mathbf{a}}\right|-1}\left(\Delta_{\mathbf{a}}\left(I^{(t)}\right) ; K\right) \\
& =\widetilde{H}^{0}\left(\Delta_{\mathbf{a}}\left(I^{(t)}\right) ; K\right) \\
& \neq(0) .
\end{aligned}
$$

Let $\mathbf{b}=k \mathbf{e}_{j}$. Then, $(\mathbf{a}+\mathbf{b})_{+}=(t-1) \mathbf{e}_{i}+(k+1) \mathbf{e}_{j}+\mathbf{e}_{p}$. Because $k+2<t$, one can check that $\Delta_{(\mathbf{a}+\mathbf{b})_{+}}\left(I^{(t)}\right)=\operatorname{star}_{\Delta}\{i\} \cup \operatorname{star}_{\Delta}\{j, p\}$ from the same argument stated above. Consequently, $\Delta_{(\mathbf{a}+\mathbf{b})_{+}}\left(I^{(t)}\right)=\Delta_{\mathbf{a}_{+}}\left(I^{(t)}\right)$. Moreover, since $G_{\mathbf{a}}=G_{\mathbf{a}+\mathbf{b}}$, we obtain

$$
\Delta_{\mathbf{a}}\left(I^{(t)}\right)=\operatorname{link}_{\Delta_{\mathbf{a}_{+}}\left(I^{(t)}\right)} G_{\mathbf{a}}=\operatorname{link}_{\Delta_{(\mathbf{a}+\mathbf{b})_{+}}\left(I^{(t)}\right)} G_{\mathbf{a}+\mathbf{b}}=\Delta_{\mathbf{a}+\mathbf{b}}\left(I^{(t)}\right) .
$$

Now, by Lemma 2.5, one has the following commutative diagram with the isomorphic map $\xi$ :

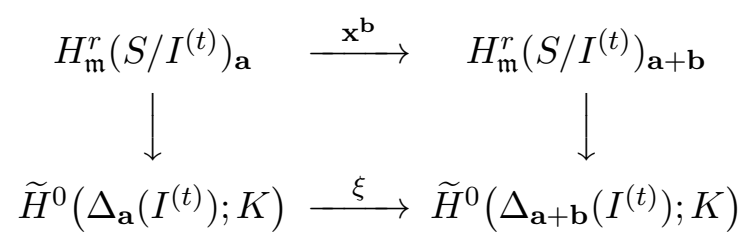

As can be seen above, $H_{\mathfrak{m}}^{r}\left(S / I^{(t)}\right) \mathbf{a} \neq(0)$; it follows that $\mathbf{x}^{\mathbf{b}} \cdot H_{\mathfrak{m}}^{r}\left(S / I^{(t)}\right) \neq$ (0). In particular, we have $\mathfrak{m}^{k} H_{\mathfrak{m}}^{r}\left(S / I^{(t)}\right) \neq(0)$, as required.

The proof of the first main result is almost finished. We now recall the statement again.

THEOREM 3.2. Let $I=I_{\Delta}$ be the Stanley-Reisner ideal of a simplicial complex $\Delta$. Let $k$ be a nonnegative integer. Then, the following statements are equivalent:

(1) $S / I^{(t)}$ is Cohen-Macaulay for all $t \geq 1$;

(2) $S / I^{(t)}$ is $k$-Buchsbaum for all $t \geq 1$;

(3) $S / I^{(t)}$ is $k$-Buchsbaum for some $t \geq k+3$;

(4) $\Delta$ is a matroid. 
Proof. Here $(1) \Rightarrow(2)$ and $(2) \Rightarrow(3)$ are trivial, while $(4) \Rightarrow(1)$ is due to [MT2, Theorem 3.1] and [V, Theorem 2.1]. What remains is to prove that $(3) \Rightarrow(4)$. We may assume that $\Delta$ is pure by [HTT, Theorem 2.6]. Suppose that $\Delta$ is not a matroid. Then, from Proposition 3.1, it follows that $\mathfrak{m}^{k} H_{\mathfrak{m}}^{r}\left(S / I^{(t)}\right) \neq(0)$ for some $r<\operatorname{dim} S / I^{(t)}$, which implies that $S / I^{(t)}$ is not $k$-Buchsbaum.

Applying Theorem 3.2 for $k=0$ and $k=1$, we immediately get the following corollary. This is a slightly better estimation than [TT, Theorem 3.9].

Corollary 3.3. Let $I=I_{\Delta}$ be the Stanley-Reisner ideal of a simplicial complex $\Delta$. Then, the following statements are equivalent:

(1) $S / I^{(t)}$ is Cohen-Macaulay for all $t \geq 1$;

(2) $S / I^{(t)}$ is Cohen-Macaulay for some $t \geq 3$;

(3) $S / I^{(t)}$ is Buchsbaum for some $t \geq 4$;

(4) $S / I^{(t)}$ is 1-Buchsbaum for some $t \geq 4$;

(5) $\Delta$ is a matroid.

When $\Delta$ is a 1-dimensional simplicial complex, the condition for $S / I^{(2)}$ to be Cohen-Macaulay was studied in [MT1, Theorem 2.3]. Now, we will give a characterization of the Cohen-Macaulayness for $S / I^{(2)}$ in terms of the $k$-Buchsbaum property.

Proposition 3.4. Let $I=I_{\Delta}$ be the Stanley-Reisner ideal of a simplicial complex $\Delta$ with $\operatorname{dim} \Delta=1$. Let $k$ be a nonnegative integer. Then, the following statements are equivalent:

(1) $S / I^{(2)}$ is Cohen-Macaulay;

(2) $S / I^{(3)}$ is Buchsbaum;

(3) $S / I^{(k+2)}$ is $k$-Buchsbaum;

(4) $\operatorname{diam}(\Delta) \leq 2$.

Proof. The equivalence of (1) and (4) follows from [MT1, Theorem 2.3]. The equivalence of (2) and (4) follows from [MN1, Theorem 3.7]. The equivalence of (3) and (4) follows from [MN3, Theorem 1.1].

By the same argument, we have the following statement as well.

Proposition 3.5. Let $I=I_{\Delta}$ be the Stanley-Reisner ideal of a simplicial complex $\Delta$ with $\operatorname{dim} \Delta=1$. Let $k$ be a nonnegative integer. Then, the following statements are equivalent:

(1) $S / I$ is Cohen-Macaulay;

(2) $S / I^{(2)}$ is Buchsbaum; 
(3) $S / I^{(k+1)}$ is $k$-Buchsbaum;

(4) $\Delta$ is connected.

We illustrate Theorem 3.2 and Propositions 3.4 and 3.5 with the following example, which also explains that the condition on $t$ in Theorem $3.2(3)$ cannot be removed.

EXAMPLE 3.6. Let $n=5$, and let $\Delta$ be a pentagon. Then the diameter of $\Delta$ is 2 , but $\Delta$ is not a matroid. Let $I=I_{\Delta}$. Then $S / I^{(2)}$ is Cohen-Macaulay, but $S / I^{(t)}$ is not Cohen-Macaulay if $t \geq 3$. More generally, $S / I^{(t)}$ is $(t-2)$ Buchsbaum, but it is not $(t-3)$-Buchsbaum for any $t \geq 3$.

Let $G$ be a simple graph, and let $I=I(G)$ be the edge ideal of $G$. The condition for $S / I^{(t)}$ to be Cohen-Macaulay for all $t \geq 1$ was studied in [RTY, Theorem 3.6]. Combining that with our result, we get the following corollary.

Corollary 3.7. Let $I=I(G)$ be the edge ideal of a graph $G$. Let $k$ be a nonnegative integer. Then, the following statements are equivalent:

(1) $S / I^{(t)}$ is Cohen-Macaulay for all $t \geq 1$;

(2) $S / I^{(t)}$ is $k$-Buchsbaum for all $t \geq 1$;

(3) $S / I^{(t)}$ is $k$-Buchsbaum for some $t \geq k+3$;

(4) $G$ is a disjoint union of finitely many complete graphs.

\section{$\S 4$. Ordinary powers}

In this section, we discuss the residue class rings of ordinary powers of a Stanley-Reiner ideal. The following lemma may be well known, but we provide a proof for the reader's convenience.

LEMMA 4.1. Let $I$ be a monomial ideal of $S$. Let $t$ and $k$ be integers with $t>0$ and $k \geq 0$. Then, $S / I^{t}$ is $k$-Buchsbaum if and only if $S / I^{(t)}$ is $k$-Buchsbaum and $\mathfrak{m}^{k} I^{(t)} \subseteq I^{t}$. In particular, $S / I^{t}$ is Cohen-Macaulay if and only if $S / I^{(t)}$ is Cohen-Macaulay and $I^{(t)}=I^{t}$.

Proof. We consider the exact sequence

$$
0 \rightarrow I^{(t)} / I^{t} \rightarrow S / I^{t} \rightarrow S / I^{(t)} \rightarrow 0
$$

We take the long exact sequence of local cohomology modules:

$$
\cdots \rightarrow H_{\mathfrak{m}}^{i}\left(I^{(t)} / I^{t}\right) \rightarrow H_{\mathfrak{m}}^{i}\left(S / I^{t}\right) \rightarrow H_{\mathfrak{m}}^{i}\left(S / I^{(t)}\right) \rightarrow H_{\mathfrak{m}}^{i+1}\left(I^{(t)} / I^{t}\right) \rightarrow \cdots
$$


We first suppose that $\mathfrak{m}^{k} I^{(t)} \subseteq I^{t}$ and that $S / I^{(t)}$ is $k$-Buchsbaum. Then, because the length of $I^{(t)} / I^{t}$ is finite, we have isomorphisms

$$
I^{(t)} / I^{t} \cong H_{\mathfrak{m}}^{0}\left(S / I^{t}\right) \quad \text { and } \quad H_{\mathfrak{m}}^{i}\left(S / I^{t}\right) \cong H_{\mathfrak{m}}^{i}\left(S / I^{(t)}\right) \quad \text { for all } i>0 .
$$

Thus, the $k$-Buchsbaumness of $S / I^{t}$ immediately follows from that of $S / I^{(t)}$. Conversely, we suppose that $S / I^{t}$ is $k$-Buchsbaum. In particular, $S / I^{t}$ is a generalized Cohen-Macaulay ring. Then, by [SV, Lemma 2.2], we have

$$
\operatorname{Ass}_{S} I^{(t)} / I^{t} \subseteq \operatorname{Ass}_{S} S / I^{t} \subseteq \operatorname{Min} S / I^{t} \cup\{\mathfrak{m}\}=\operatorname{Min} S / I \cup\{\mathfrak{m}\}
$$

while it follows that $I^{(t)} S_{P}=P^{t} S_{P}=I^{t} S_{P}$ for any $P \in \operatorname{Min} S / I$ from the definition of symbolic powers. Thus, it follows that the length of $I^{(t)} / I^{t}$ is finite. Again using the isomorphisms of local cohomology modules stated above, we get $\mathfrak{m}^{k} I^{(t)} \subseteq I^{t}$ and the $k$-Buchsbaumness of $S / I^{(t)}$.

When $k=0$, the Cohen-Macaulay case follows.

Lemma 4.2. Let $I=I_{\Delta}$ be the Stanley-Reisner ideal of a simplicial complex $\Delta$ with $\operatorname{dim} \Delta>0$. If $S / I^{(t)}$ is a $k$-Buchsbaum ring for some $t>k \geq 0$, then $\Delta$ is connected.

Proof. Note that $\Delta$ is pure by [HTT, Theorem 2.6]. Let $\mathbf{b}=k \mathbf{e}_{1}$. Then, $\Delta_{\mathbf{b}}\left(I^{(t)}\right)=\Delta_{\mathbf{0}}\left(I^{(t)}\right)=\Delta$. Indeed, $F \in \operatorname{Max}\left(\Delta_{\mathbf{b}}\left(I^{(t)}\right)\right)$ if and only if $\sigma_{F}^{\mathbf{b}}<t$ and $F \in \operatorname{Max}(\Delta)$ by Lemma 2.3. Thus, the equality $\Delta_{\mathbf{b}}\left(I^{(t)}\right)=\Delta$ follows. For the same reason, we have the equality $\Delta_{\mathbf{0}}\left(I^{(t)}\right)=\Delta$. Now, by Lemma 2.5 , the following commutative diagram follows, where $\xi$ is an isomorphism:

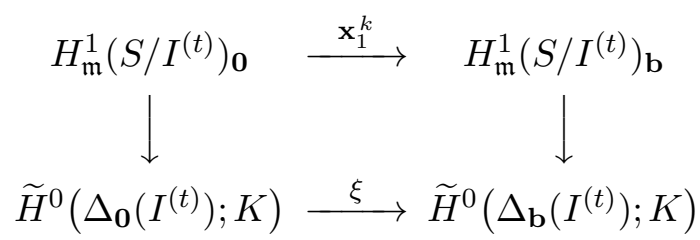

On the other hand, $\xi$ is a zero map since $S / I^{(t)}$ is $k$-Buchsbaum with $\operatorname{dim} S / I^{(t)} \geq 2$. Thus, $\widetilde{H}^{0}(\Delta ; K)=(0)$. Hence, $\Delta$ is connected.

Before presenting the proof of the results of this section, we recall an important result due to Terai and Trung. We note that the simplicial complex $\Delta$ is called a complete intersection if its Stanley-Reisner ideal $I_{\Delta}$ is a complete intersection. 
TheOREM 4.3 ([TT, Theorem 4.5]). Let $I=I_{\Delta}$ be the Stanley-Reisner ideal of a simplicial complex $\Delta$ with $\operatorname{dim} \Delta \geq 2$. Then, the following statements are equivalent:

(1) $S / I^{t}$ is generalized Cohen-Macaulay for all $t \geq 1$;

(2) $S / I^{t}$ is generalized Cohen-Macaulay for some $t \geq 3$;

(3) $\Delta$ is a disjoint union of finitely many complete intersection complexes of the same dimension.

First we state the result with $\operatorname{dim} \Delta \geq 2$, as follows.

THEOREM 4.4. Let $I=I_{\Delta}$ be the Stanley-Reisner ideal of a simplicial complex $\Delta$ with $\operatorname{dim} \Delta \geq 2$. Let $k$ be a nonnegative integer. Then, the following statements are equivalent:

(1) $S / I^{t}$ is Cohen-Macaulay for all $t \geq 1$;

(2) $S / I^{t}$ is $k$-Buchsbaum for all $t \geq 1$;

(3) $S / I^{t}$ is $k$-Buchsbaum for some $t \geq \max \{3, k+1\}$;

(4) $I$ is a complete intersection.

Proof. It is enough to check the implication $(3) \Rightarrow(4)$. Because $S / I^{t}$ is a generalized Cohen-Macaulay ring, the conclusion follows from Lemmas 4.1 and 4.2 and Theorem 4.3.

Finally, we state the results of a 1-dimensional case. A simplicial complex with $\operatorname{dim} \Delta=1$ can be regarded as a simple graph. We say that $\Delta$ is $n$-path (resp., $n$-cycle) if $\Delta$ is a path of $n+1$ vertices (resp., a cycle of $n$ vertices).

Theorem 4.5 ([TT, Theorem 4.4]). Let $I=I_{\Delta}$ be the Stanley-Reisner ideal of a simplicial complex $\Delta$ with $\operatorname{dim} \Delta=1$. Then, the following statements are equivalent:

(1) $S / I^{t}$ is generalized Cohen-Macaulay for all $t \geq 1$;

(2) $S / I^{t}$ is generalized Cohen-Macaulay for some $t \geq 3$;

(3) $\Delta$ is a disjoint union of paths or cycles.

THEOREM 4.6. Let $I=I_{\Delta}$ be the Stanley-Reisner ideal of a simplicial complex $\Delta$ with $\operatorname{dim} \Delta=1$. Assume that the number of vertices of $\Delta$ is at least three. Let $k$ be a nonnegative integer. Then, the following statements are equivalent:

(1) $S / I^{t}$ is Cohen-Macaulay for all $t \geq 1$;

(2) $S / I^{t}$ is $k$-Buchsbaum for all $t \geq 1$;

(3) $S / I^{t}$ is $k$-Buchsbaum for some $t \geq k+3$; 
(4) I is a complete intersection;

(5) $\Delta$ is a 2-path, a 3-cycle, or a 4-cycle.

Proof. The equivalence between (1) and (5) follows from [MT1, Corollary 3.5]. The implications $(1) \Rightarrow(2) \Rightarrow(3)$ are trivial. Suppose that condition (3) is satisfied. Then, $S / I^{(t)}$ is $k$-Buchsbaum for some $t \geq k+3$ by Lemma 4.1. Hence, $\Delta$ is a matroid by Theorem 3.2. On the other hand, by Theorem $4.5, \Delta$ is a disjoint union of paths or cycles. Thus, $\Delta$ is a path or a cycle because a matroid must be connected. (This follows from Lemma 4.2, too.) Using the characterization for a graph to be a matroid in [TT, Corollary 2.6], one can see that $\Delta$ should be a 2-path, a 3-cycle, or a 4-cycle as in (5). The equivalence between (4) and (5) is easy to check.

Combining the cases of $\operatorname{dim} \Delta=1$ and $\operatorname{dim} \Delta \geq 2$, we get the following.

Corollary 4.7. Let $I=I_{\Delta}$ be the Stanley-Reisner ideal of a simplicial complex $\Delta$ with $\operatorname{dim} \Delta>0$. Let $k$ be a nonnegative integer. Then, the following statements are equivalent:

(1) $S / I^{t}$ is Cohen-Macaulay for all $t \geq 1$;

(2) $S / I^{t}$ is $k$-Buchsbaum for all $t \geq 1$;

(3) $S / I^{t}$ is $k$-Buchsbaum for some $t \geq k+3$;

(4) I is a complete intersection.

When $\Delta$ is a 1-dimensional simplicial complex, the condition for $S / I^{2}$ to be Cohen-Macaulay was studied in [MT1, Theorem 3.4]. We give a characterization of the Cohen-Macaulayness for $S / I^{2}$ in terms of the $k$-Buchsbaum property.

Proposition 4.8. Let $I=I_{\Delta}$ be the Stanley-Reisner ideal of a simplicial complex $\Delta$ with $\operatorname{dim} \Delta=1$. Assume that the number of vertices of $\Delta$ is at least three. Let $k$ be a nonnegative integer. Then, the following statements are equivalent:

(1) $S / I^{2}$ is Cohen-Macaulay;

(2) $S / I^{3}$ is Buchsbaum;

(3) $S / I^{k+2}$ is $k$-Buchsbaum;

(4) $\Delta$ is a 2-path, a 3-cycle, a 4-cycle, or a 5-cycle.

Proof. The equivalence of (1), (2), and (4) follows from [MT1, Corollary 3.4] and [MN2, Theorem 4.10]. The implication $(1) \Rightarrow(3)$ follows from Proposition 3.4 and Lemma 4.1. The remaining part is the implication that $(3) \Rightarrow(4)$. We may assume that $k>0$. By Lemma $4.2, \Delta$ is connected, and 
by Theorem $4.5, \Delta$ is a path or a cycle. On the other hand, by Lemma 4.1, $S / I^{(k+2)}$ is $k$-Buchsbaum, whence it follows that $\operatorname{diam}(\Delta) \leq 2$ from Proposition 3.4. Consequently, $\Delta$ is a path or a cycle with $\operatorname{diam}(\Delta) \leq 2$; thus $\Delta$ should be a 2-path, a 3-cycle, a 4-cycle, or a 5-cycle.

We illustrate Theorem 4.6 and Proposition 4.8 with the following example, which also explains that condition (3) in Theorem 4.6 is optimal.

EXAMPLE 4.9. Let $n=5$, and let $\Delta$ be a 5 -cycle. Let $I=I_{\Delta}$. Then $S / I^{2}$ is Cohen-Macaulay, and $S / I^{3}$ is not Cohen-Macaulay but is Buchsbaum. More generally, $S / I^{t}$ is not $(t-3)$-Buchsbaum for any $t \geq 4$.

Acknowledgments. The authors are grateful to the referees for careful readings and valuable advice, especially concerning a generalization in our original result using the concept of a matroid. The research for this article was carried out while the first author visited Meiji University, and he thanks that institution for its hospitality and support.

\section{REFERENCES}

[BH] W. Bruns and J. Herzog, Cohen-Macaulay Rings, rev. ed., Cambridge University Press, Cambridge, 1998. MR 1251956.

[CN] R. C. Cowsik and M. V. Nori, On the fibres of blowing up, J. Indian Math. Soc. (N.S.) 40 (1976), 217-222. MR 0572990.

[GH] D. H. Giang and L. T. Hoa, On local cohomology of a tetrahedral curve, Acta Math. Vietnam. 35 (2010), 229-241. MR 2731325.

[GT] S. Goto and Y. Takayama, Stanley-Reisner ideals whose powers have finite length cohomologies, Proc. Amer. Math. Soc. 135 (2007), 2355-2364. MR 2302556. DOI 10.1090/S0002-9939-07-08795-3.

[HTT] J. Herzog, Y. Takayama, and N. Terai, On the radical of a monomial ideal, Arch. Math. (Basel) 85 (2005), 397-408. MR 2181769. DOI 10.1007/s00013-005-1385-z.

[M] N. C. Minh, Cohen-Macaulayness of ideals associated with graphs, Ph.D. dissertation, Meiji University, Kawasaki, Japan, 2009.

[MN1] N. C. Minh and Y. Nakamura, The Buchsbaum property of symbolic powers of Stanley-Reisner ideals of dimension 1, J. Pure Appl. Algebra 215 (2011), 161167. MR 2720681. DOI 10.1016/j.jpaa.2010.04.007.

[MN2] - Buchsbaumness of ordinary powers of two-dimensional square-free monomial ideals, J. Algebra 327 (2011), 292-306. MR 2746039. DOI 10.1016/j.jalgebra. 2010.07.027.

[MN3] - A note on the $k$-Buchsbaum property of symbolic powers of StanleyReisner ideals, Tokyo J. Math. 34 (2011), 221-227. MR 2866644. DOI 10.3836/ $\mathrm{tjm} / 1313074452$.

[MT1] N. C. Minh and N. V. Trung, Cohen-Macaulayness of powers of two-dimensional square-free monomial ideals, J. Algebra 322 (2009), 4219-4227. MR 2558862. DOI 10.1016/j.jalgebra.2009.09.014. 
[MT2] Cohen-Macaulayness of monomial ideals and symbolic powers of StanleyReisner ideals, Adv. Math. 226 (2011), 1285-1306. MR 2737785. DOI 10.1016/j. aim.2010.08.005.

[RTY] G. Rinaldo, N. Terai, and K.-I. Yoshida, Cohen-Macaulayness for symbolic power ideals of edge ideals, J. Algebra 347 (2011), 1-22. MR 2846393. DOI 10.1016/j. jalgebra.2011.09.007.

[S] A. Schrijver, Combinatorial Optimization, Polyhedra and Efficiency, Vol. C: Disjoint Paths, Hypergraphs, Chapters 70-83, Algorithms Combin. 24,C, Springer, Berlin, 2003. MR 1956926.

[SV] J. Stückrad and W. Vogel, Buchsbaum Rings and Applications: An Interaction between Algebra, Geometry and Topology, Springer, Berlin, 1986. MR 0881220.

[T] Y. Takayama, Combinatorial characterizations of generalized Cohen-Macaulay monomial ideals, Bull. Math. Soc. Sci. Math. Roumanie (N.S.) 48(96) (2005), 327-344. MR 2165349.

[TT] N. Terai and N. V. Trung, Cohen-Macaulayness of large powers of Stanley-Reisner ideals, Adv. Math. 229 (2012), 711-730. MR 2855076. DOI 10.1016/j.aim.2011. 10.004 .

[TY] N. Terai and K.-I. Yoshida, Locally complete intersection Stanley-Reisner ideals, Illinois J. Math. 53 (2009), 413-429. MR 2594636.

[V] M. Varbaro, Symbolic powers and matroids, Proc. Amer. Math. Soc. 139 (2011), 2357-2366. MR 2784800. DOI 10.1090/S0002-9939-2010-10685-8.

Nguyên Công Minh

Department of Mathematics

Hanoi National University of Education

Hanoi

Vietnam

minhnc@hnue.edu .vn

Yukio Nakamura

Department of Mathematics

School of Science and Technology

Meiji University

Kawasaki-shi 214-8571

Japan

ynakamu@meiji.ac.jp 